\title{
Underwater Resurrection Routing Synergy using Astucious Energy Pods
}

\author{
Shahzad Ashraf ${ }^{1}$, Asif Raza ${ }^{2}$, Zeeshan Aslam ${ }^{3}$, Hamad Naeem ${ }^{4}$, Tauqeer Ahmed ${ }^{5}$ \\ ${ }^{1,5}$ College of Internet of Things Engineering, Hohai University, Changzhou, Jiangsu, China \\ ${ }^{2}$ Department of Automation, Shanghai Jiao Tong University, Shanghai, China \\ ${ }^{3}$ Department of HSE, Petroweld Oilfield Services, Kurdistan Region, Iraq \\ ${ }^{4}$ College of Computer Science, Neijiang Normal University, Neijiang, Sichuan, China \\ 1'nfc.iet@ hotmail.com, ${ }^{2}$ asifraza151@sjtu.edu.cn, ${ }^{3}$ zeeshan.bhutta0078@gmail.com, ${ }^{4}$ hamadnaeemh @ yahoo.com, \\ 5tauqeer_rao@hotmail.com
}

\begin{abstract}
The accomplishment of sustainable communication among source and destination sink node is a rigors challenge and even establishing bodacious communication link between these nodes is nothing short of a miracle because data routes are governed by the underwater environment. Energy consumption has a significant influence as all active devices rely on the battery. As cost-effective data packet transmission is established as a norm, no charging or replacement can be achieved. Hop link evaluation and shrewd connection discovery by way of a resurrecting linking element were just a genuinely grim task, and only feasible to create the extra powered energy pods (URRSAEP) that had never been carried out before after detailed study. After packet transfer, the sensor node performs the link inspection process, and when a link is deemed shaky at less than or equivalent to 50 percent of capacity, the target node incorporates its residual capacity status and returns it to the source node that attaches other unoptimizable energy pods to improve only the targeted node link from 50 percent to 90 percent. Performance evaluation using NS2 with Aqua-Sim 2.0 simulator has been obtained comparing with DBR and EEDBR protocols in terms of point-to-point delay, Packet dissemination ratio, Network lifespan and Energy Diminution.
\end{abstract}

Keywords- Underwater routing, hop links, ramshackle, energy pods, resurrect link factor, end-to-end delay, network performance

\section{INTRODUCTION}

Acoustic communication is usually defined as the method of sending and receiving message using sound emitted in the subsequent underwater environment. Underwater sensing networks include a variety of vehicles and sensors that work together to track and collect data in a particular area. UWSNs establish large-scale operations to track large areas of the sea. But the fulfillment of that dream depends on effective and secure protocols and underwater networking facilities. Sadly, the comprehensive expertise gained over several decades of research on the development of radio-frequency-based Wireless Sensor Networks (WSNs) cannot be focused on the design of UWSN networking protocols. The underwater acoustic system's basic characteristics, such as long-run latency and low bandwidth transmission, and aquatic ecosystem physical factors including salinity and seabed sediment are responsible for this. Nonetheless, the architecture of networking protocols for UWSNs poses numerous new problems not even seen in standard WSNs. The usage of the underwater acoustic network entails increased bandwidth, high and increasing propagation period, transient route loss, high vibration, multipath loss, shadow zones, Doppler propagation and high transmission energy costs. In addition, wireless underwater communications suffer from extreme time variations, as fluctuations in water temperature are disrupted that change the sound refraction and the seabed sediment [1].

While optical signals are safe, however, radio frequency (RF) signals require higher attenuation in permeable water but encounter the adsorption problem. It obstructs acoustic signals such as reducing bandwidth, bits failure rate, and latency count [2]. In particular, UWSNs are used in the fields of oil and gas discovery, battlefields for surveillance, building inspections, imagination targeting, catastrophe detection and preventative action, submarine targeting, exploitation of natural and offshore resources, Identification of ambient conditions including variations in atmosphere, illumination, sound or unusual objects etc. [3].

Simultaneously, UWSN faces unchallenging obstacles. Sensor node is completely battery-dependent and it is challenging to replace or replace batteries in a brawny environment [4], whereas there is little possibility of taping solar energy due to rapid dynamic change in the water surface. Furthermore, acoustic transmissions are subject to greater distance propagation that engulf an enormous amount of energy compared to terrestrial network. Therefore, only detour remaining to build an astucious routing by which the data packet may traverse the sink node from origin to target surface and eventually avoid the energy loss. Researchers have function out for establishing a specialist routing 
mechanism to achieve the desired performance and thereon developed several energy efficient routing protocols.

Direct packet forwarding between source and surface sink node is difficult to enforce, since this process causes infinite waste of energy. Researchers have therefore adopted the opportunistic routing based methodology involving inappropriate data flooding thereon every node transmits packets sheaf, called flooding, that consumes excessive energy while locating the pathway. Same like, OR uses endto-end delay, packet transmission, etc. to discover the suitable neighboring relay node. Though under some conditions it works perfectly, causing packet duplication that involves exorbitant energy wastage on time. A basic cognitive solution is spatial routing, that does not set up a whole route but putting in the location information for the packet to be sent. Same like, each hop node near the destination transmits the packet, but there is a large risk of void fistula that are likely to harm the entire mechanism.

There are two classes of underwater routing protocols, location based and location free. First, taking into account location based protocols, GPS plays a key role and offers network position details with the help of the sink, but major problems occur when the importance of local routing is diminished by the irregular environment. At the same time, location free routing logs have greater capability but are also inappropriate to use, for example, network parameters that don't search efficiently for a next forwarder node, and the possibility of an inappropriate selection of the links that would consume high energy [5]. The suggested URR-SAEP underwater routing synergy, however, using residual energy which would not impact the next forwarder's connection aspect, nor is it troubled by depth results. Nevertheless, DBR has a better risk of wasting energy when selecting the standard passage because of shaky connections.

Underwater nodes may die faster in the normal routing scheme when they are under low water pressure. In order to address the above essential problems, a reliable underwater routing technique needs to be built to absorb negligible energy and achieve the necessary results. Routing link factor plays a decisive role in underwater results, and typically researchers rely on conventional link estimators instead of a revived linking trend with substantial explanations for improving packet routing.

Contributions to analysis is being summarized as.

In order to expand an energy efficient route with the use of energy pods, the Shrewd Underwater Routing Coordination (URR-SAEP) aims. It is a calm energy storage system running in three stages, taking into account the following:

- Resurrect link factor;

- Depth and residual energy;

- Packet transmission.
The resurrected connection is a special inspection of hop connection. The hop connection factor after getting data when a sensor node transmits packets to neighbours, activates the linking inspection mechanism as defined in information flow chart Figure 1. When the connection threshold is determined to be equal to or greater than $50 \%$ of energy pods, the accepting node will recognize by adding residual energy details to the received packet and sending it back to the reference node. If significant information is obtained, the source node again sends only this node to the duplicate packet and this time, the duplicate packet has an extra energy pods, which improves connection capacity at the very least from 50 percent to 90 percent. A successful packet transfer is now performed and hence relay node creation is likely to be completed. The entire approach has been discussed in the Methodology section. This conception of the resurrect link element in other ground research has not yet been studied.

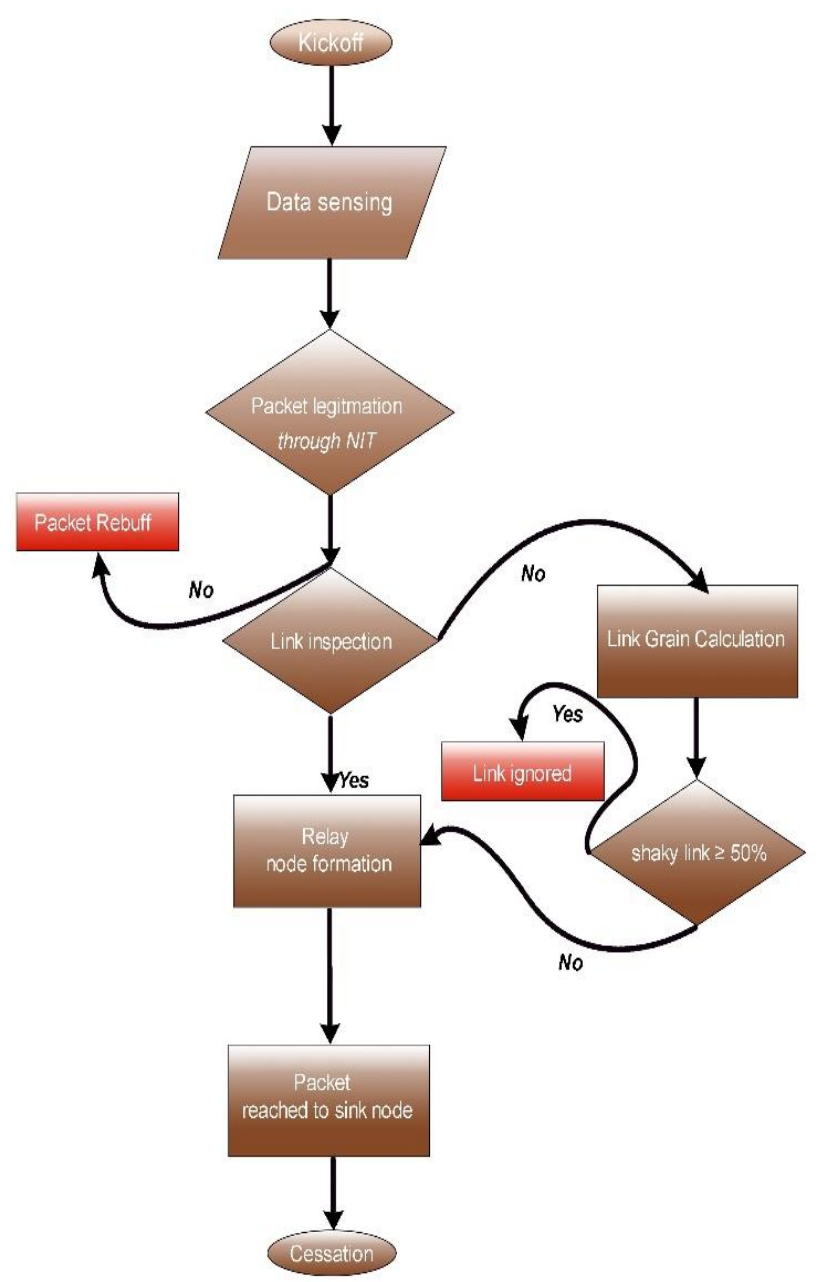

Fig. 1. Proposed (URR-SAEP) information process mechanism

The rest of the findings are structured as follows: Section II highlights the relevant study, section III provides information about the suggested technique for routing (URRSAEP), section IV discusses performance assessment using 
simulation tests, and section $\mathrm{V}$ presents the observations and possible future research directions.

\section{RELATED WORK}

Acoustic communication is, due to the special channel design, the only quiet option for underwater data routing [6]. Radio waves and electromagnetic waves are ideal mediums for terrestrial communication, Because it occupies a broad range of spaces but these fail absolutely in the case of underwater, thereupon in such situation, acoustic signals play the desired function, in view of the confined bandwidth and a rather lengthy speed of propagation around $1500 \mathrm{~m} /$ $\mathrm{s}$ [7]. The nodes with vitriol water pressure vanish quickly in the normal approach.

In comparison to the radio frequency, the acoustic signal starts the transmission cycle through a single medium. While some disgusting carriers cause energy leakage, the acoustic signal is reflected, scattered and absorbed by the surface of the seabed and water and the transmitting data is thus lost. The underwater acoustic signal works at frequencies from $10 \mathrm{~Hz}$ to $1 \mathrm{MHz}$. Just a small number of frequencies are used in underwater communications due to a narrow acoustic spectrum [8]. Inevitable variables as salinity, temperature and depth of water only affect the amplitude of the acoustic signal, the acoustic wave travels along the curved path, and the sensor nodes do not overhear the signals. This is also likely to establish a void region and Nodes in such an area cannot be involved in the transmission cycle, that ultimately contributes to the existence of the network.

Battery replenishing or restoring in the underwater environment is not an simple job, especially in severe environments, and so an effective data routing protocol that can manage and sustain the routing direction from both the ground and the surface is necessary to distribute the data packets at the required level with minimal power.

As the protocol for energy-efficient underwater transport is developed, other unrivaled obstacles such as its restricted bandwidth, which foresees high power usage and even losses in transmission route in the case of long-range transmission. In addition to the fact that there are several routing protocols in service which are believed to be power effective but need a separate routing route at every point during the transmission, the acoustic signal speed is often more sluggish as well [9]. In reality they are inefficient and consume exorbitant resources. Therefore, communication performance is wasted energy by hollow link transmission, most routing strategies tend to take into account. The related underwater opportunistic (OR) protocols are evaluated on the basis of its class structure.

(A). Location based Opportunistic Routing: Using sensor node position information, OR generates a $3 \mathrm{D}$ imaginary virtual tube between transmitters and sink node to prevent FSR selection problem. A Fixed Combination Technique (FRC) Energy Capture / Routing Scheme (ARCUN) was proposed [10], to improve the intelligent use of energy usage with the loading of monitored data packets without the implementation of medium access monitoring systems. The findings indicate that this strategy places additional data transmission loads on relay nodes that shortens the lifetime of the network.

Considering direction-based routing by Ahmed, S., et al. [11], each node knows its position at the position of its neighbours in a single hop and also records the location of the sink Node. The relation quality specifies the flooding area from source to sink node in order to forward a packet. Although this approach results in an unclosed parcel delivery relationship with negligible packet load, no steps are taken to tackle void cases, so in a sparse setting it is not suitable.

Vijayalakshmi, P. et al [12], developed a stateless routing protocol to join a continuous and interlaced path between the source node and the sink node, thus requiring just a few nodes in the process of transition. A self-adapting algorithm preserves the redundant paths and Nodes can be choosing the right path to transfer the packet. When it receives the packet, the destination node calculates the direction and reports the distance from the forwarder by the adjacent arrival angle (AOA). It is favorable for smaller networks but uncouth for multi-settings, given the severity of its packet loss and node failure.

Khasawneh, A. et al [13], proposed a pressured locationfree underwater (RE-PBR) protocol, mainly take the relation consistency, information and residual energy into account. The triangle approach was used to investigate the connection quality and the multimetric transfer algorithm was developed to estimate the route costs. This approach is only suited small small networks and lack of void handling.

Hoa Tran [14] developed a cooperative routing technology (USASNs) through utilizing a cross-layer setup merged the MAC and the network layer for improved communication. The data have been transmitted with the routing relay technologies while linked to cooperative relays. In terms of factors including SNR and arrival time, the source node sends a packet. This definition appears to be obvious, but it may operate in a linear setting, although the packet supply chain will be decreased as the medium state, in deep water or in shallow water is not defined. Moreover, without placing the findings into the void field, which appears to be a shrewd strategy.

(B). Location Free Opportunistic Routing: Based on the number of hop counts, the suburb nodes are identified using complex address and pressure information. The sink node delivers promptly the beacon messages that passes with a specific identity known as a dynamic address from the surface to the inner depth. The distinct Network Topologies with the same information-like addresses allocated to the sensor nodes are used for common beacon related protocols. Because OR uses topological details to find 
transmission relay nodes, remove FSR by using $2 \mathrm{H}-\mathrm{ACK}$ [15], every node is restricted to a complex address such as a beacon post, the neighboring node with the smaller address and closer to the sink, and the forwarding node is chosen. The only next forwarder node not a placate approach is taken into account for the purpose of resolving the DFS issue in case of insecure link.

The impact of forwarding node has been proactively analyzed with angle based power efficient mechanism proposed by Ashraf, $S$ et al [16], carrying out the packet transmission by considering the forwarder hop angle (FHA) and the Counterpart Hop Angle (CHA). A three-state link quality has doped out the targeted forwarding relay node among the neighboring nodes which analyzed how much batteries are draining out by the forwarder node when a packet has been transmitted. Three-state link quality metrics is adopted with predefined parameters and utilizing the Additive-Rise and Additive-Fall method which increases the probability of packet collision. Therefore, no proper impact measures are addressed for this fistula.

Wang, Z. et al. (EAVARP) [17] recorded a combated energy-aware and void-aware routing scheme where concentration shells have been built around the sink node and sensor nodes have been positioned in such shells dynamically. Furthermore, the OR directional forwarding scheme was implemented (ODFS) in which data packs with a residual amount of energy inside the same shell were forwarded that circumvented every vacuum area. While the writers proposed a smart solution, they did not adopt the energy wasting scheme that eventually shortened the lifetime of the network.

The decentralized and routing table-based strategy SOSRP [18], Implemented that lists the total number of hops from the source to the sink node. Routes were self-structured and therefore unused nodes were isolated. Such an initiative is suitable only for a minimal network and cannot track excessive packet pollution. There is no control over energy usage, and nodes tend to expire sooner than scheduled. Each node uses maximum available energy for packets transmition. Following each round of transmission, nodes restructure the routes which eventually raises energy costs. Of the location-routing sequence, the void problem was solved with location-routing by Barbeau, M., et al [19]. The location of the next node of transmitting hope depends on: $(i)$ the number of hopes, (ii) route and (iii) the state of scope. A beacon alert updates the routing information is used to navigate the route between sink and source node. The pressure control tool needs higher power consumption to calculate the path [20]. Table 1. provides a description of the stringent comparison of the current (URR-SAEP) approach with other rival protocols.

Table 1. Comparative study of methods suggested for other UW routing schemes

\begin{tabular}{|c|c|c|c|c|c|}
\hline Protocol & Principle Area & Working Ground & Expediency & Impairments & Proposal (URR-SAEP) \\
\hline $\begin{array}{l}\text { DBR by } \\
\text { Costantino, G., } \\
\text { et al. [3] }\end{array}$ & Depth information & $\begin{array}{l}\text { Greedy routing } \\
\text { technique }\end{array}$ & $\begin{array}{l}\text { Lowest holding } \\
\text { time, better packet } \\
\text { distribution ratio }\end{array}$ & $\begin{array}{l}\text { Energy swindler, } \\
\text { t high end to end } \\
\text { delay, void holes, } \\
\text { packet duplicate }\end{array}$ & $\begin{array}{l}\text { Limited energyusage, trivial } \\
\text { end-to-end delay, better void } \\
\text { handling }\end{array}$ \\
\hline $\begin{array}{l}\text { DFR by } \\
\text { Ahmed, S., et } \\
\text { al. [11] }\end{array}$ & $\begin{array}{l}\text { Distance and quality of } \\
\text { connection (ETX) }\end{array}$ & $\begin{array}{l}\text { Location aware } \\
(\text { Own }+ \text { next hop } \\
\text { neighbor }+ \text { sink } \\
\text { node })\end{array}$ & $\begin{array}{l}\text { Reliable Packet } \\
\text { delivery, petty } \\
\text { overhead }\end{array}$ & $\begin{array}{l}\text { Inoperative void } \\
\text { field event for the } \\
\text { parsing network }\end{array}$ & $\begin{array}{l}\text { Suitable for dense } \\
\text { and spars network } \\
\text { as well }\end{array}$ \\
\hline $\begin{array}{l}\text { VBF by } \\
\text { Vijayalakshmi, } \\
\text { P., et al. [12] }\end{array}$ & Distance details & $\begin{array}{l}\text { Passage interchangeable } \\
\text { and interlaced, } \\
\text { minimum distance to the } \\
\text { sink inside pipeline }\end{array}$ & Robust, scalable & $\begin{array}{l}\text { Multi-sink } \\
\text { bottleneck } \\
\text { condition }\end{array}$ & $\begin{array}{l}\text { The choice of connection } \\
\text { performance tolerates the } \\
\text { situation of the bottleneck }\end{array}$ \\
\hline $\begin{array}{l}\text { RE - PBR by } \\
\text { Khasawneh, } \\
\text { A., et al. [13] }\end{array}$ & Triangle metric & $\begin{array}{l}\text { Multi - metric route } \\
\text { cost }\end{array}$ & $\begin{array}{l}\text { Performance } \\
\text { correlation }\end{array}$ & $\begin{array}{l}\text { Cannot handle } \\
\text { void nodes }\end{array}$ & $\begin{array}{l}\text { Intelligently stop unnecessary } \\
\text { communication }\end{array}$ \\
\hline $\begin{array}{l}\text { UW - ASNs by } \\
\text { Tran - Dang et } \\
\text { al. [14] }\end{array}$ & $\begin{array}{l}\text { Cooperate } \\
\text { routing }\end{array}$ & $\begin{array}{l}\text { MAC and network layer } \\
\text { of cross-section } \\
\text { architecture }\end{array}$ & $\begin{array}{l}\text { Link quality and } \\
\text { network overhead }\end{array}$ & $\begin{array}{l}\text { Uncouth energy } \\
\text { wastage }\end{array}$ & $\begin{array}{l}\text { Shrewd energy } \\
\text { utilization }\end{array}$ \\
\hline $\begin{array}{l}2 \mathrm{H}-\mathrm{ACK} \text { by } \\
\mathrm{Wu}, \mathrm{H} ., \text { et al. } \\
{[15]}\end{array}$ & $\begin{array}{l}\text { Dynamic node } \\
\text { address }\end{array}$ & $\begin{array}{l}\text { Nodes near to sink } \\
\text { are prioritized }\end{array}$ & $\begin{array}{l}\text { Confined } \\
\text { overhead }\end{array}$ & $\begin{array}{l}\text { Unreliable link } \\
\text { with single } \\
\text { forwarder }\end{array}$ & $\begin{array}{l}\text { Multi-forwarder atmosphere, } \\
\text { selection of links is related to } \\
\text { measurement of relation grains }\end{array}$ \\
\hline $\begin{array}{l}\text { SOSRP by } \\
\text { Hindu, S., [18] }\end{array}$ & Decentralized & $\begin{array}{l}\text { Self - organized } \\
\text { routes }\end{array}$ & $\begin{array}{l}\text { Continent for } \\
\text { smaller } \\
\text { network }\end{array}$ & $\begin{array}{l}\text { Energy intake } \\
\text { unregulated }\end{array}$ & Impediment to energy loss \\
\hline $\begin{array}{l}\text { EEDBR by } \\
\text { Wahid, A. [27] }\end{array}$ & $\begin{array}{l}\text { Depth and residual } \\
\text { energy }\end{array}$ & $\begin{array}{l}\text { Sender node links } \\
\text { forwarder }\end{array}$ & $\begin{array}{l}\text { Confine energy } \\
\text { consumption }\end{array}$ & $\begin{array}{l}\text { Uncouth packet } \\
\text { dissemination }\end{array}$ & $\begin{array}{l}\text { Bodacious packet } \\
\text { delivery }\end{array}$ \\
\hline
\end{tabular}




\section{METHODOLOGY}

A detailed analysis was carried out to achieve a reliable and efficient underwater mechanism that revealed the concept of Underwater Resurrection Routing Synergy using Astucious Energy Pods (URR-SAEP). The selection of a sagacious connection and packet forwarding mechanism has been extensively investigated. The forwarding relay node formation is shown in Figure 2.

\section{A. Operational Model}

The suggested network architecture (URR-SAEP) primarily includes sensor nodes used at various depths of which the sink node on the top of the water interacts with the offshore base station. The sink node receives data from both the source and neighboring relay nodes. This is compatible with both RF and acoustic modems. The acoustic modem is used to communicate with the sensor nodes mounted under water while the RF modem is used to relay data to the base station. A good data packet gets across intermediate neighboring relay nodes by hop-by-hop routing rovers to the surface sink. The node receives the accurate details through the depth sensor when the remaining energy is dispensed. The path to the closest relay node has been ratified by Received Signal Strength (RSS) at the receiving node [21] and the attenuation of the signal is based on spread losses calculated by Thorp formula. The absorption loss $\alpha(f)$ is calculated in Eq. (1), for a certain frequency $f$ :

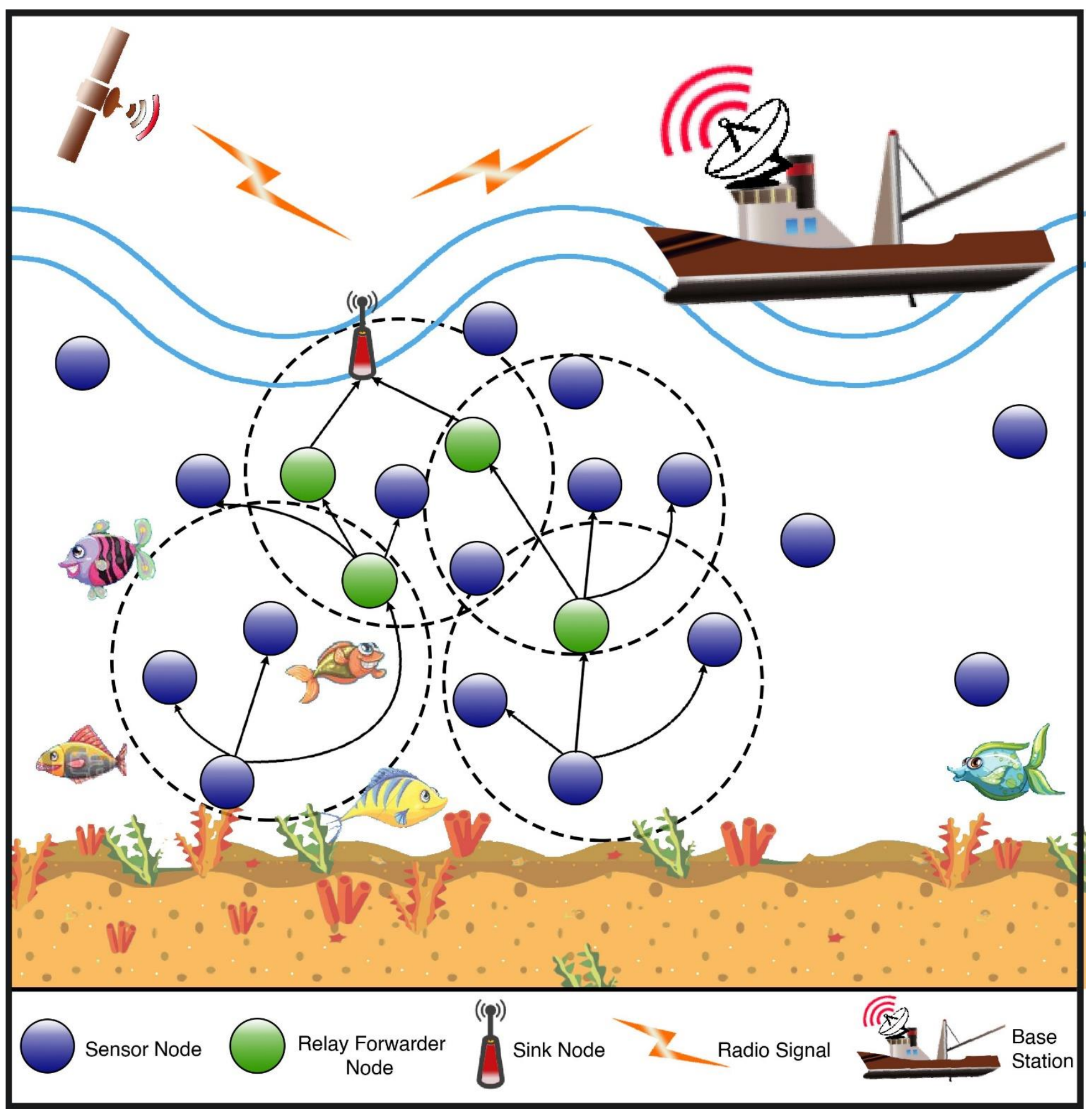

Fig. 2 Proposed URR-SAEP network topology 


$$
10 \log (\alpha(f))= \begin{cases}\frac{0.11 f^{2}}{1+f^{2}}+\frac{44 f^{2}}{(4100+f)}+2.75 \times 104 f^{2}+0.003, & f \geq 0.4 \\ 0.002+0.11\left(\frac{f}{(1+f)}\right)+0.011 f, & f<0.4\end{cases}
$$

here $\alpha(f)$ counts $d B=k m$, while is marked by $f$ and $\alpha$ becomes parallel with absorption loss thereby listed as $\alpha=\frac{10^{\alpha(f)}}{10}$. An average loss count yield attenuation factor as $\mathrm{A}(1, f)$ and spreading can be computed by Eq.(2).

$$
10 \log (A(l, f))=k \times 10 \log l+l \times 10 \log (\alpha(f))
$$

obtained the spreading loss as $k \times \log l$ possessed distance $l$ while the absorption $\operatorname{loss} l \times 10 \log (\alpha(f))$ and given $k$ as coefficient factor shows signal scattering geometry.

\section{B. Link Factor}

The energy utilization, data dissipation frequency and system performance is relying on robustness of the connection. Indeed, the protocol based on the expected transmission count (ETX) [22], measures the consistency of the communication between two directions of each path during the transfer process. These protocols use GPS locational information or gather finite data from the sink point, while suggested technique (URR-SAEP) decides the link level in its best way by taking some step. Although some other link estimation methods like cost-based routing are in use, the exponentially weighted average window mean (WMEWMA) [23], determines the memory-efficient path estimator. A number of packets are needed to control the amount of packets transmitted and retransmitted before an effective transmission during the expected time (RNP) [24]. The demanded packet number (PRR) [25] is a receipt-side connection estimator dependent on the time window shift. Both of these interaction estimators have inoperative restrictions, and a clear correlation has not been successfully established. The proposed analysis (URR-SAEP) was precisely carried out on a single dimension to calculate the mere relationship factor:

Step 1: Link factor indicator (LFI) and signal-to noise ratio (SNR) leverage.

For instance $n$, taking as absolute broadcasting packets, whereas $m$ has taken into account positive packets, while $i$ points the packets obtained successfully by calculating their contact efficiency by means of the $i$, lfi and snri measurement (link factor indicator). In view of the hardware-based connection element, evaluate whether the relation has an appropriate range of quality then the node is entered in the table next step. The SNR compares the transmitted signal amplitude with the source sound and measures the signal ratio. The combination of $l f i$ and snri is indicative of the probability of re-vitalizing the LIF and SNR higher values.

Step 2: LFI and SNR mean calculation.

There are inherent limits for an aggregate standard. It cannot take the packet loss, so still monitors the nodes it has gotten. Connecting value metric from $(0,0)$ to $(\overline{S N R}, \overline{L F I}$ update the link factor. On the basis of $l f i$, snri and PRR, the mean $(\overline{S N R}, \overline{L F I})$ is computed by PRR metric and thereby avoids the statistical mean. The filled values are provided as Eq. (3) and (4).

$$
\begin{aligned}
& \overline{S N R}_{w}=\frac{\sum_{k=1}^{m} s n r_{k}}{n} \\
& \overline{L F I}_{w}=\frac{\sum_{k=1}^{m} l f i_{k}}{n}
\end{aligned}
$$

Step 3: Distance measurement

Taking origin $(0,0)$ state and the point $(\overline{S N R}, \overline{L F I})$ link factor can be identified by calculating the path $d \Delta$ from this point, thereby Eq. (5) yields

$$
d \Delta=\sqrt{\overline{S N R}_{w}{ }^{2}-\overline{L F I}_{w}{ }^{2}}
$$

\section{Step 4: Sagacious route}

While the lengthiest path from source to neighboring nodes $d \Delta$ believes that it is the highest quality of the link but not a pure eligible connection, a predefined value of th threshold will reveal the connection factor between both, as expressed in Eq. (6).

$\Psi=\left\{\begin{array}{l}\text { Shrewd link, } \\ \text { Pristine link, } \\ \text { Fair link, } \\ \text { Uncouth link, }\end{array}\right.$

$$
\begin{aligned}
& t h_{\text {shrewd }}<d \Delta \\
& t h_{\text {pristine }} \geq d \Delta<t h_{\text {shrewd }}(6) \\
& t h_{\text {fair }} \geq d \Delta<t h_{\text {pristine }} \\
& d \Delta<t h_{\text {uncouth }}
\end{aligned}
$$

The recommended mechanism (URR-SAEP) is centered on the metric triangle (TM) [26], thus evaluating the reliability of the connection from source to neighboring node, and maintaining a Link Repository Table (LRT). Table 2. shows the threshold parameters on which the link factor is calculated.

Table 2. Links and threshold value

\begin{tabular}{lcccc}
\hline Metric type & SNR & LFI & PRR & Triangle \\
\hline Shrewd link & $>30$ & $>106$ & 1 & $>145$ \\
Pristine link & $15-30$ & $102-106$ & $0.75-1$ & $80-145$ \\
Fair link & $5-15$ & $80-102$ & $0.35-0.75$ & $30-80$ \\
Uncouth link & $0-5$ & $0-80$ & $0-0.35$ & $0-30$ \\
\hline
\end{tabular}

\section{Link Factor Information Gathering Cycle}

Increasing sensor node gathers information from lowerthan-owned neighboring nodes and sends a hello response within the transmission range possessed ID, distance, and the residual energy. When this message is received, A defined pattern is followed in each node and the (NIT) information is 
submitted to ratify the eligibility of the message and allowed if its depth is greater, then the message would not be admitted. Next, the consistency of the connections is analyzed by measuring the cumulative values of SNR, LFI, and PRR. The sequence of estimating starts as a sensor node transmits the ID, SNR, and LFI value specimen packets. PRR produces mean values in the next step, while the relation continuity is determined by distance calculation dependent on the TM values. The last step adjusts a NIT chart by joining the node range while the target point of a low end node is calculated. Algorithm 1. ensures the order of acquisition of information and therefore communicates its dissemination as follows:

Step1: Every sensor node (nodea) generates a HELLO (CreateHELLO) message and sends it to nearby nodes.

Step2: Every adjacent node gets the message (GetHELLOW) and follows the appropriate steps.

Step3: The NIT table constantly updates information about nodes due to frequent positions changes.

\begin{tabular}{|c|c|}
\hline \multicolumn{2}{|c|}{ Algorithm 1. Retrieving Data } \\
\hline 1: & Procedure CreateHello(node $a)$ \\
\hline 2: & if HelloLatency is disappeared then \\
\hline 3: & Create Hellopacket \\
\hline 4: & $\begin{array}{l}\text { Include } i d \text {, depth, and residual energy in } \\
\text { HelloPacket }\end{array}$ \\
\hline 5: & Broadcast (HelloPacket) \\
\hline 6: & Set NewInterval \\
\hline 7: & endif \\
\hline 8: & endprocedure \\
\hline \multicolumn{2}{|l|}{ 9: } \\
\hline 10: & Procedure GetHello(nodea, HelloPacket) \\
\hline 11: & if $\mid$ node $\boldsymbol{a}$. depth $|>|$ HelloPacket.depth $\mid$ then \\
\hline 12: & $\begin{array}{l}\text { if HelloPacket id is invalid in nodea.NIT } \\
\text { then }\end{array}$ \\
\hline 13: & $\begin{array}{l}\text { Include HelloPacket information to } \\
\text { nodea.NIT }\end{array}$ \\
\hline 14: & else \\
\hline 15: & update information in node $\boldsymbol{a} . \boldsymbol{N I T}$ \\
\hline 16: & endif \\
\hline 17: & goto link factor triangle metric(nodea) \\
\hline 18: & else \\
\hline 19: & rebuff(HelloPacket) \\
\hline 20: & endif \\
\hline 21: & endprocedure \\
\hline
\end{tabular}

\section{Packet Forwarding and Route Cost}

Packet headers tend to pass from source to target sink entities, and all nodes are completely active in the packet forwarding process but only if nearest forwarding node is close to the destination sink node with better connectivity and more residual energy available. However, Wahid, A [27], estimated a minimal cost element, Remaining energy or ETX dependent. Yet we use residual energy to calculate route costs and thus measure the distance dependent on the TM. From now on, the metric of the path between the two nodes, i.e. $(x$, $y$ ), is calculated as expressed in Eq. (7).

$$
\text { Route Cost }(x, y)=\left(1-\frac{\operatorname{Res}_{y}}{\operatorname{Res}_{\max }}\right)+\left(1-\frac{\Delta d_{(x, y)}}{\Delta d_{\max }}\right)
$$

here $\operatorname{Res}_{y}$ is a residual energy of node $y$, while energy of all nodes has been represented by $\operatorname{Res}_{\max }$, whereas $\Delta d_{\max }$ is a system parameter. The connection quality parameters of source and forwarder hubs has been gotten as $\Delta d_{(x, y)}$. Considering two variable factors like, residual energy and efficient communication link, using Eq. (7) route cost can be determined. Calculation shows that node must be less insightful than source node and therefore pristine communication efficiency is a result preserving route costs at minimum. To pick the next forwarding node as illustrated in Figure 3, source node, $a$, captures surrounding nodes' credentials from NIT. Proceeding to next step, route cost can be determined by Eq. (7). The lower cost node of the route is chosen therefore $b$ will be selected. Algorithm 2. specifically executes a logical forwarding method for packets.

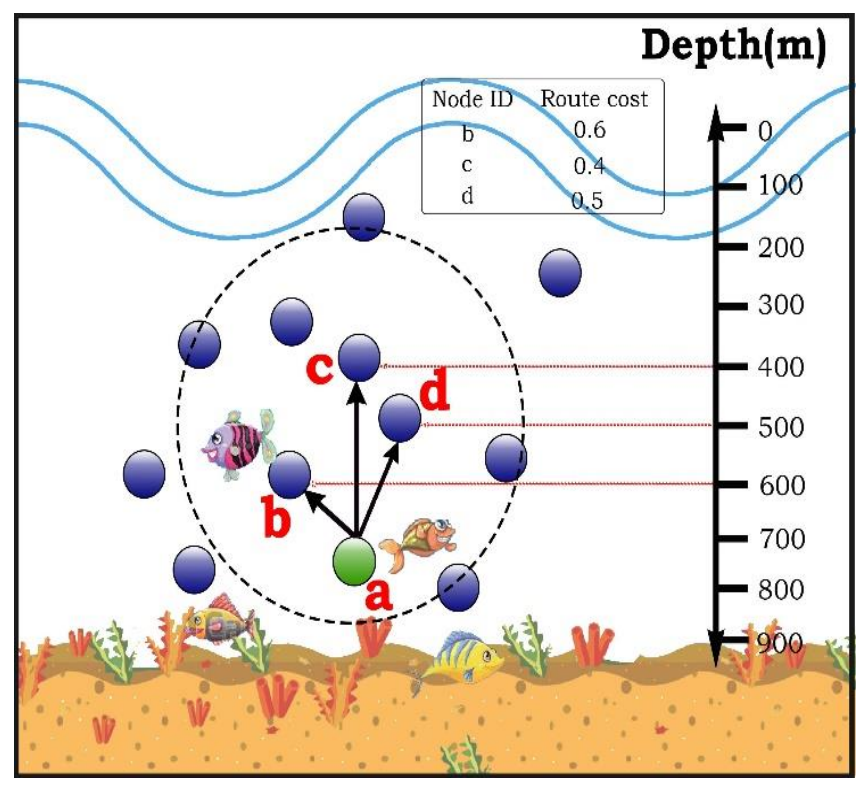

Fig. 3 Relay forwarding of the node selection process

The source node $a$ accepts data packet ID and moves to the next-hop neighbor. The packet ID fits the transmitting node ID at the transmission node and if the packet is deemed valid it refuses the packets otherwise. Eventually, the data packet enters the destination sink node by reiterating the same operation. The data packet may be hurdled to the final destination due to uncouth UWSNs [26]; the packet travels through multiple regions and may malfunction anywhere. 


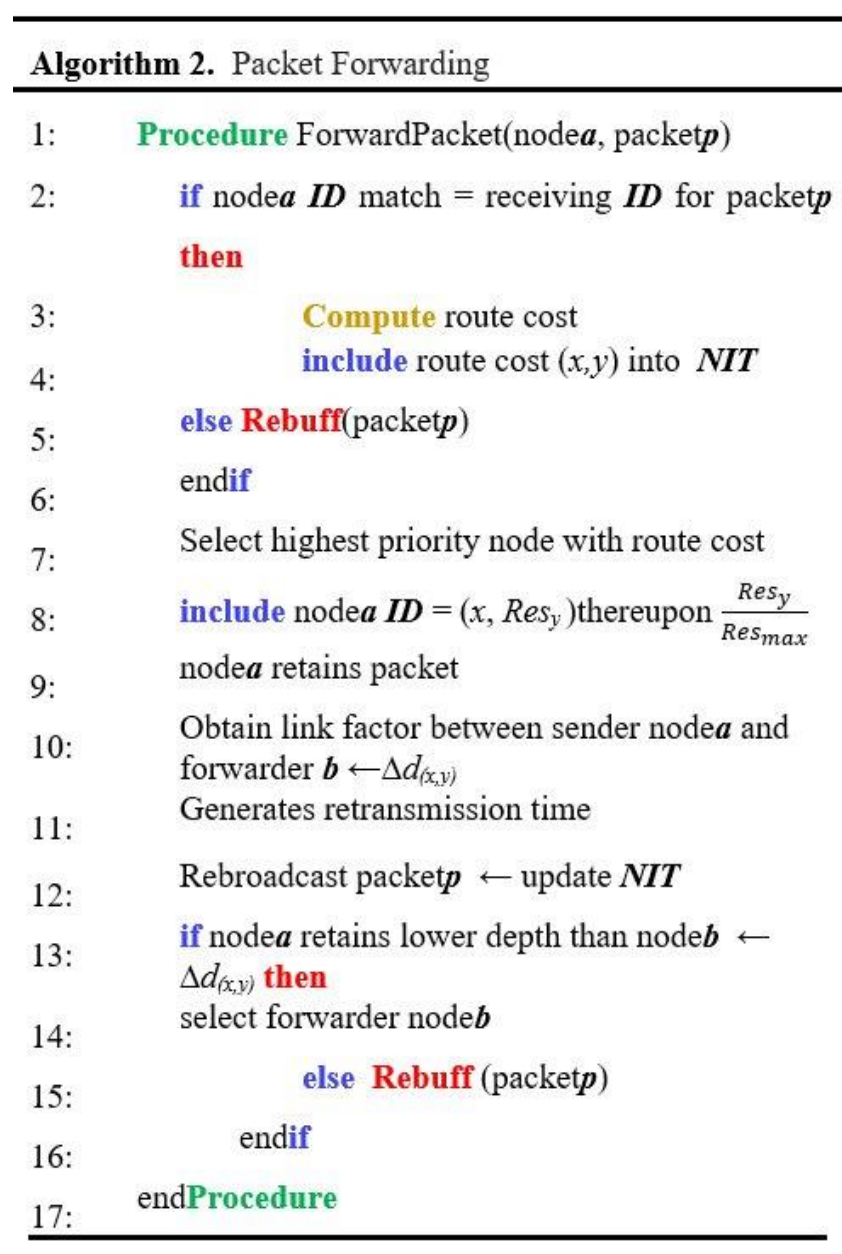

\section{E. Link Grain Calculation}

The suggested method measures and preserves more acceptable standard of the link and strengthens the principle of reparation of the link. The packet $\mathrm{p}$ is being transmitted by the sensor node a, encompassing credentials like distant information, residual energy and ID either towards the surrounding nodes like $a, b$ and $c$ depicted in Figure 4. For example, among $N$ series of source node, the node $\mathrm{Na}$ transmits packet towards surrounding nodes and thereby node

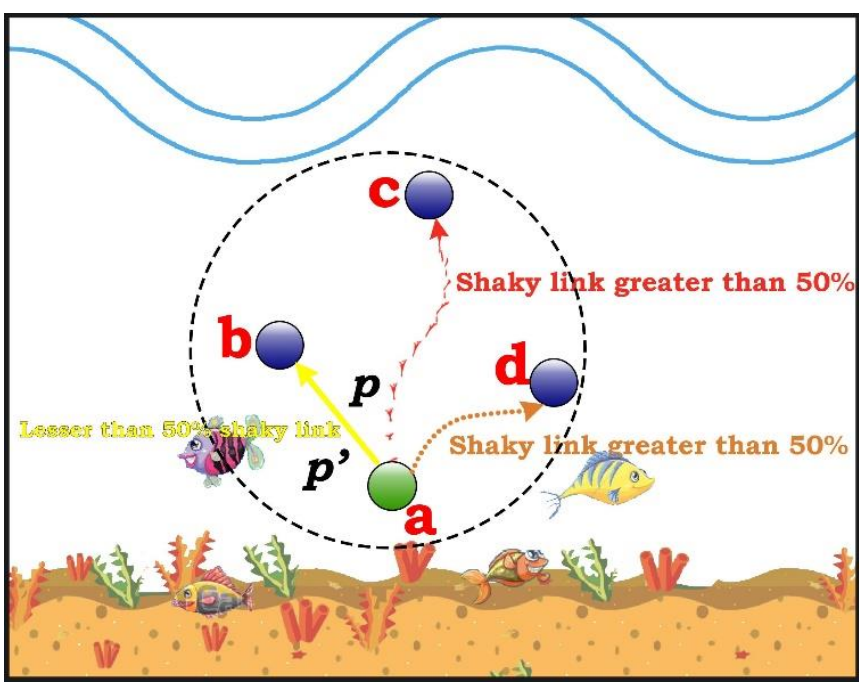

Fig. 4 Link grain determination $b$ acknowledges this packet and incorporates essential details as $N b p$ and reverberate to source node $a$.

This time incorporating substantial energy pods and duplicating node $a$, thereby sending packet again towards node $b$ as $N a_{2 p}$ in negligible time $t$, and computes the link grain as expressed in Eq. (8).

$$
\text { Link Grain }=N a_{p}+N b_{p^{\prime}}+N a_{2 p},
$$

Eventually, efficiency of the connection is improved with the $E a_{p}, E b_{p^{\prime}}$ and $E a_{2 p}$, energy consumption, which stays unaltered respectively, and thus Eq. (9) changes the likelihood of the relation from 50 to 90 percent in due course.

Link Grain $=t\left(\sum_{p=1}^{N a_{p}} E a_{p}+\sum_{p^{\prime}=1}^{N b_{p \prime}} E b_{p^{\prime}}+\sum_{2 p=1}^{N a_{2 p}} E a_{2 p}\right)$,

There are identified connection connections between node $a$ and other nodes. A mandated inspection of the link consistency is performed which determines that hop links are more over 50 percent dilapidated and that links are much more stable than 50 percent. For instance, the consistency of the link between the source node $a$ and $b$ was more than $50 \%$ solid not just more than $90 \%$ stable, thus the links between the source node $a$ to $c$ and $d$ are now more than $50 \%$ fragile. The hop relationship in both the node $\mathrm{a}$ and $\mathrm{b}$ made it safer to achieve a smooth packet transmission, i.e., up to 90 percent.

\section{PERFORMANCE EVALUATION}

Through comparing the suggested solution with the DBR and EEDBR protocols and to use the NS2 simulator, namely Aqua-Sim, the performance has been extensively analyzed. The simulation configuration parameters have been implemented as defined in Table 3. For this assessment, nodes between 100-400 and approximately 1000 x $1000 \mathrm{x}$ $900 \mathrm{~m}^{3}$ area and $100 \mathrm{~m}$ distance between each sensor is considered. While simulation begins thereby adjacent node overhears residual energy and takes the depth information in the hi-packet interval, i.e., $99 \mathrm{~s}$, a distance-based TM is

Table 3. Simulation specifications

\begin{tabular}{ll}
\hline Parameter & Value \\
\hline Deployment area & 1000 x $1000 \times 900 \mathrm{~m}^{3}$ \\
Distance among sensor couplet & $100 \mathrm{~m}$ \\
No. of nodes & {$[100-600]$} \\
Communication range & $250 \mathrm{~m}$ \\
Type of protocol & SMAC \\
Start energy & $100 \mathrm{~J}$ \\
Medium & Acoustic Waves \\
Bandwidth capacity & $10 \mathrm{Kbps}$ \\
Packet generation rate & $0.02 \mathrm{pkts} / \mathrm{min}$ \\
Velocity & $1500 \mathrm{~m} / \mathrm{s}$ \\
Node movement & $0-3 \mathrm{~m} / \mathrm{s}$ \\
Energy consumption & $2 \mathrm{~W} ; 0.75 \mathrm{~W} ; 8 \mathrm{~mW}$ \\
Data packet volume & $64 \mathrm{bytes}$ \\
Data packet interval (Hello) & $99 \mathrm{~s}$ \\
Packet creation time & $15 \mathrm{~s}$ \\
No. of runs & 50 \\
\hline
\end{tabular}


estimated, and as the basic case, the energy model [28], also calculates waste energy and energy usage. After 99 transmissions we just found half and provided the tests.

\section{A. Point-to-Point delay}

A total duration or time period is called point-to-point delay for packet passes across various zones and acknowledged by destination sink. Unwanted barriers to forwarding, scattering and data processing caused to slow down the packet transmission. Eq. (10), assesses the delay point-to-point. When acknowledging at final destination, the entire packet corpus is defined as the $l^{\text {th }}$ simulation, $B T_{l, m}$

Point to point Impediment $=\frac{\sum_{l=1}^{50} \sum_{m=1}^{P C}\left(B T_{l, m}-A T_{l, m}\right)}{P C \times 50}$

indicates $m^{\text {th }}$ packet's broadcast time. Similarly, at destination, $A T_{l, m}$ is the time of acceptance of the $m^{\text {th }}$ packet.

During underwater routing, the key explanation for packet keeping time is the point-to-point delay avoided during the planned URR-SAEP. Figure 5, confirms regarding delay is found trivial than of rival protocols. It also ratified that these situations will be same in dense and sparse environment, despite been countless calculations during transmission processes.

Holding packet duration and reliance on depth knowledge causes significant challenges for DBR and it could not perform well as compares to proposed URR-SAEP system. In comparison, a marginal point-to-point delay is found for EEDBR relative to DBR, as it employed a method for retaining residual energy-based packets but, when a sudden failure occurs, it increases even the retaining time, which may result in infinite delay.

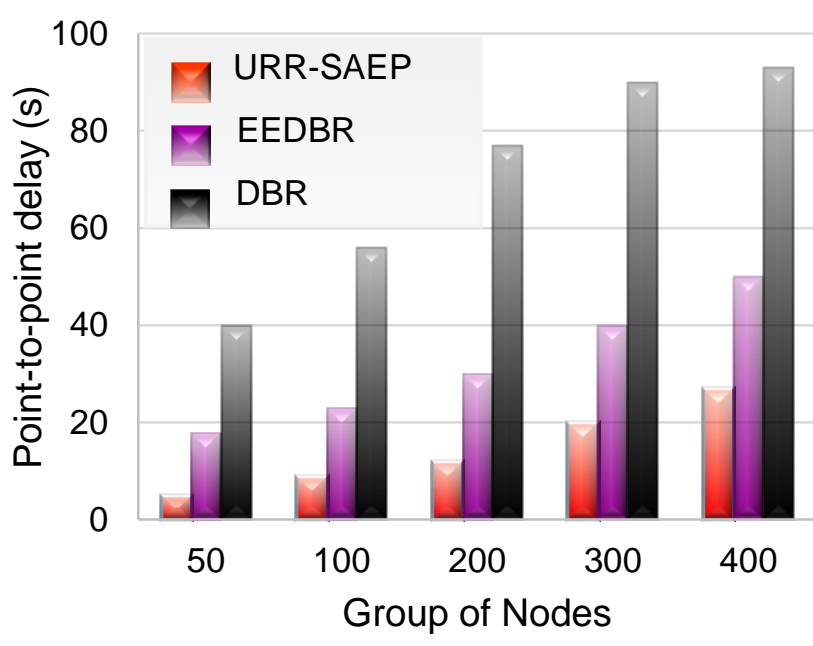

Fig. 5 Output of point to point packet delay
A numerical association between the transmitted packets is regarded as the delivery ratio of the packets when it is obtained at the targeted destination node in some shape or measurements. Mainly, defined in Eq. (11), this relationship (PDR) is expressed in percentage.

$$
P D R \%=\left\{\left(\sum_{n=1}^{50} \frac{P B}{P R}\right) / 50\right\} \times 100
$$

here $P B$ and $P R$ determine the ratio of packet transmitted and receiving during the nth simulation.

A shrewd packet output is conceivable when large nodes are included. Although this assertion is better suited to DBR, there is ample adjustments in the packet distribution, a packet period provides extra transmission that will enhance packet conflict, i.e., sparse to dense if the network switches status. With astute selection and higher residual capacity, the unparalleled packet transmission frequency from URR-SAEP is rendered possible. The suggested scheme obtained an increased distribution ratio of around 12 and 18 percent in approximately 160 nodes compared with those of DBR and EEDBR according to the Figure 6. As the number of nodes exceeded by 500 when ending the transmission, the URRSAEP received an unbeatable ranking of 22 and $12 \%$ relative at most to DBR and EEDBR.

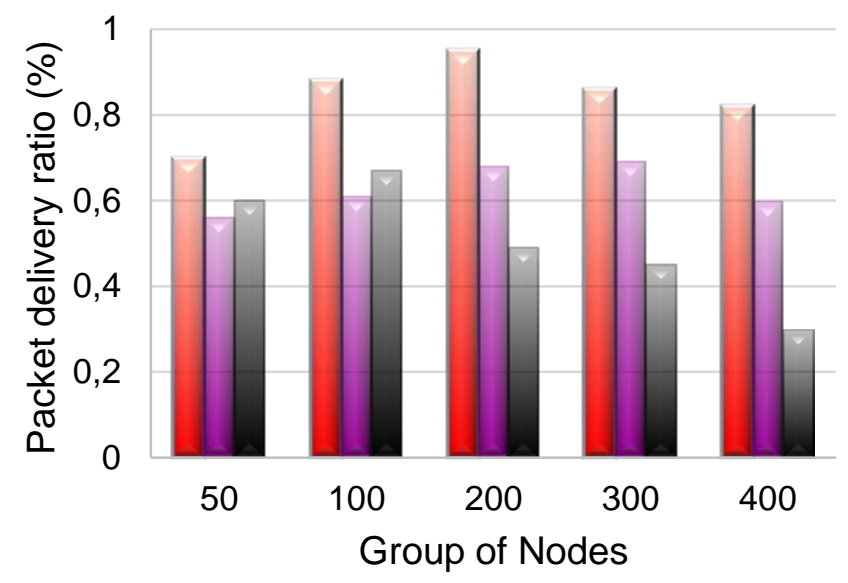

Fig. 6 Overall packet delivery ratio to sink node

\section{Network Lifespan}

It's streamlined network life, sensor nodes constantly submit and receive packets. Two methods, i.e., range-based and scheduling-based, are used to attain a longer life cycle. A schedule-based methodology, which was defined by the SMAC Protocol [29], for the simulation results was used in the proposed URR-SAEP process. A whole network lifetime contained the period from the first transmission until the destination node and the network contribution of the last packet is verified. Network lifecycles can be determined with Eq. (12).

\section{B. Packet dissemination ratio}




$$
\text { Network Lifespan } \quad=\quad \frac{\sum_{l=1}^{50}\left(N N T_{l}-N H T_{l}\right)}{50}
$$

where network adjust at $N N T_{l}$ intervals for $l^{\text {th }}$ simulation, thereupon stops at $N H T_{l}$ moment. The factors indicate that when the nodes and area range is increased, the lifespan is thereby shrieked. Nevertheless, if transmission power between different rates can be balanced shrewdly by sensor nodes and packet load can therefore be distributed across all S-MAC nodes, network reliability can be further revitalized.

Therefore, in accordance to the result obtained in Figure 7, the URR-SAEP system was superior to its adversaries i.e., DBR and EEDBR. During the forwarding of URR-SAEP, greater residual power with a shrewd connection factor allowed for extending the network lifespan. Furthermore, no packet holding tangle was included in the proposed system, so there was no redundant packet transmission impediment, irrespective of how often dense or sparse the network volume was. The network has thus gained stability, which could potentially prolong its life. In fact, the lifetime of DBR was shorter for the transmission and the residual energy was not consumed except in the depth information only used to pick the transmission node. Therefore, nodes which reside under shallow water cannot remain in existence for higher and therefore expire quickly, resulting the vacillating network lifetime. Atmospheric energy and depth information are worth the shrewd usage compared to DBR. Thus, it was only verified that the number of nodes were capable of engaging in packet forwarding, so that no further redundant packets remained in the tests. Nevertheless, the EEDBR's output did not reach the proposed URR-SAEP scheme even closely.

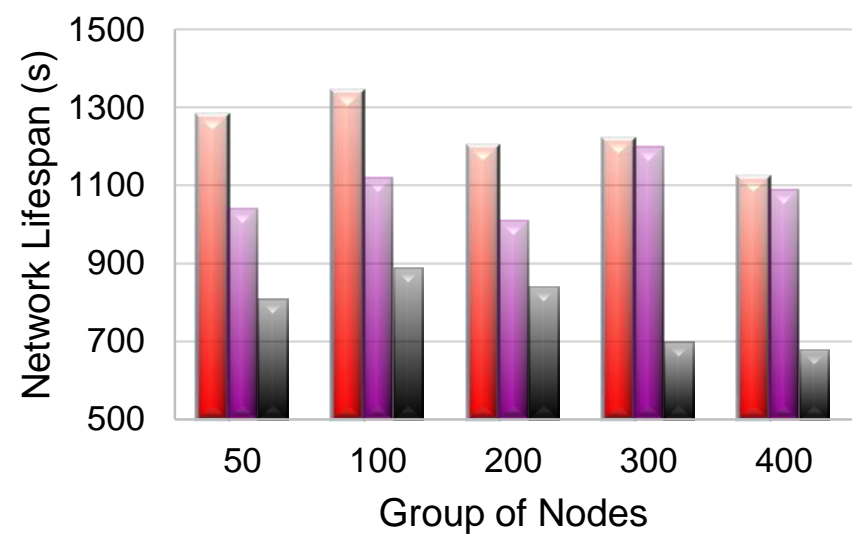

Fig. 7 Duration when network remained active

\section{Energy Diminution}

During the transmission round, all nodes have average energy consumption to send packets towards destination sink. Eq. (13), is being used for all node to assess the power consumption. A node therefore uses $E_{x}$ energy, while beacon message transmits $p$-bits over displacement $d$, from now on:

$$
E_{x}(p, d)=\left\{\begin{array}{c}
p \cdot E_{d s}+p \cdot E_{f s . d^{2}} \\
p . E_{d s}+p \cdot E_{m p \cdot d^{4}}
\end{array}\right.
$$

when $p . E_{d s}$ is a debasement of the signal, $p . E_{f s}$; indicates free space while $p . E_{m p}$ is a multipath scattering. The sensor node collects the energy $p$-bits set, thereby consuming the $E_{e}$ energy defined by Eq. (14),

$$
E_{p}(p)=p \cdot E_{d s}
$$

for the forwarder relay node, the $E_{f}(p, d)$ potency is released in Eq. (15), while the amount of energy used by the sensor node during packet forwarding has been marked by $E_{f}$,

$E_{f}(p, d)=p \cdot E_{d s}+p \cdot E_{f s}=\left\{\begin{array}{c}2 p \cdot E_{d s}+p \cdot E_{f s \cdot d^{2}} \\ 2 p \cdot E_{d s}+p \cdot E_{m p \cdot d^{4}}\end{array}\right.$

for the energy phase, the packet consuming an entire energy when reaching at destination sink node has specifically been vouched by Eq. (16), which summarizes the final and pristine output.

$$
E_{f}(p, d)=p \cdot E_{d s}+p \cdot E_{f s}
$$

the suggested URR-SAEP approach provides the desired results illustrated in Figure 8. In comparison with DBR and EEDBR, it consumed only marginal energy throughout the whole time. This positive outcome was definitely obtained even because the reliable delivery of the packet has been impeded. In fact, the use of energy has been rendered more from the intelligent aspects of residual energy and relation efficiency. This method was not effective in all phases so URR-SAEP was initially faced with such a crisis, with a significantly higher energy usage level so soon improvements were made to the forklift node and an appropriate general usage. The energy consumption ratio is exponential at roughly 290 to 600 nodes, ratified that paths are stable while packet failure is almost vacillated, therefore, whole packets returning towards destination sink node preventing additional energy pods. In contrast with the EEDBR and DBR, the performance is between 27 and $32 \%$ respectively, a significant achievement in this case.

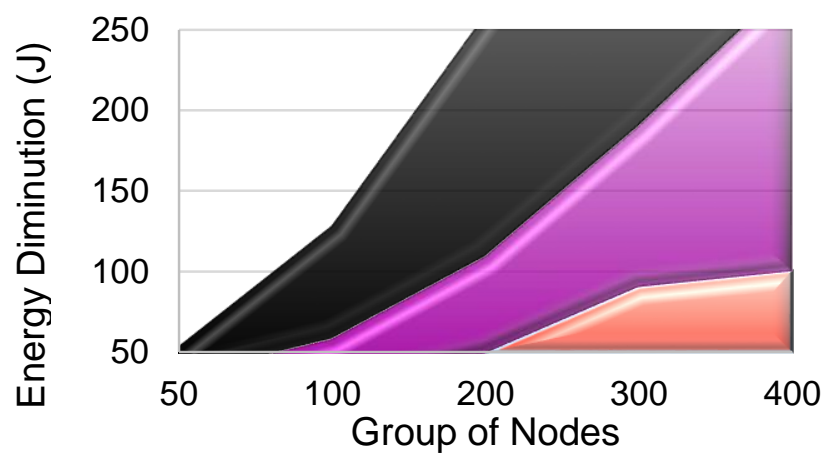

Fig. 8 Overall energy used by whole network 


\section{E. Performance Review}

The point-to-point data limitation prevents the hold time for the packet triggering a late attempt to relay the packet. DBR and EEDBR are unabled to work well well as comparing to the suggested URR-SAEP approach due to packets retaining time and residual energy. For packet delivery ratio, the time needed for packet holds for DBR and EEDBR while the network state switches either dense or sparse. This may potentially increase the packet conflict and the network bottleneck. URR-SAEP achieved bodacious performance of 22 and 12 per cent compared to DBR and EEDBR respectively when it reached the end of the transmission period. The use of the S-MAC protocol not only made the network's lifetime longer, but also faced greater energy utilization. The sensor nodes altered the energy transfer for matching the packet load across network, thus denying the duplicate packet transfer. DBR and EEDBR transmission has long been unstable and generated worthless output.

\section{CONCLUSION AND FUTURE DIRECTIONS}

Just astute protocols will allow the network viable for underwater routing. The function of batteries therefore is essential and critical since the whole transmission depends on battery, and the results are obtained if shrewd protocols use the small battery capacity wisely. URR-SAEP's proposed strategy was the same as expected at design time. Applying restore relationships with residual energy and depth data made it easy to select the next most appropriate forwarding point, unlike other traditional approaches. Comprehensive outcomes, which were never anticipated by conventional routing schemes, are obtained in terms of point-to-point delay, packet payout size, network life and energy reduction Revitalizing the performance of the link by the measurement of grain from the origin is a novel concept that considers the correlation between the source and the neighboring nodes and analyses the related values as a reversal between less than $50 \%$ and more than $50 \%$ separately. In the case a connection is shaky close to or under $50 \%$, a source node attaches an additional energy shield, takes into consideration the remaining energy of the goal node and shrews the relation up to $90 \%$. We expect a further smart artificial intelligence analysis to optimize the usage of UWSN bandwidth through segmental allocation strategy to create an all-inclusive surveillance scalable network.

\section{REFERENCES}

[1] Guan, Q., Ji, F., Liu, Y., Yu, H., \& Chen, W. (2019). Distance-VectorBased Opportunistic Routing for Underwater Acoustic Sensor Networks. IEEE Internet of Things Journal, 6(2), 3831-3839. doi:10.1109/jiot.2019.2891910

[2] Su, Y., Fan, R., Fu, X., \& Jin, Z. (2019). DQELR: An Adaptive Deep Q-Network-Based Energy- and Latency-Aware Routing Protocol
Design for Underwater Acoustic Sensor Networks. IEEE Access, 7, 9091-9104. doi:10.1109/access.2019.2891590

[3] Ashraf, S., Gao, M., Chen, Z., Kamran, S., \& Raza, Z. (2017). Efficient Node Monitoring Mechanism in WSN using Contikimac Protocol. International Journal of Advanced Computer Science and Applications, 8(11). doi:10.14569/ijacsa.2017.081152

[4] Qu, J., Zhang, Z., Cui, Y., Wang, J., \& Mastorakis, G. (2019). Research and Application of Multi-Node Communication and Energy Consumption Prediction Control in Underwater Acoustic Network. IEEE Access, 7, 41220-41229. doi:10.1109/access.2019.2907376

[5] Ashraf, S., Ahmed, T., Raza, A., \& Naeem, H. (2020). Design of Shrewd Underwater Routing Synergy Using Porous Energy Shells. Smart Cities, 3(1), 74-92. https://doi.org/10.3390/smartcities3010005

[6] Tilwari, V., Maheswar, R., Jayarajan, P., Sundararajan, T. V. P., Hindia, M. N., Dimyati, K., Amiri, I. S. (2020). MCLMR: A Multicriteria Based Multipath Routing in the Mobile Ad Hoc Networks. Wireless Personal Communications. https://doi.org/10.1007/s11277-02007159-8

[7] Qureshi, U., Shaikh, F., Aziz, Z., Shah, S., Sheikh, A., Felemban, E., \& Qaisar, S. (2016). RF Path and Absorption Loss Estimation for Underwater Wireless Sensor Networks in Different Water $\begin{array}{lll}\text { Environments. } & \text { Sensors, } & 16(6),\end{array}$ https://doi.org/10.3390/s16060890

[8] Somani, Arun K., et al. Smart Systems and IoT: Innovations in Computing: Proceeding of SSIC 2019. Springer, 2020.

[9] Coutinho, R. W. L., Boukerche, A., Vieira, L. F. M., \& Loureiro, A. A. F. (2018). Underwater Wireless Sensor Networks. ACM Computing Surveys, 51(1), 1-36. https://doi.org/10.1145/3154834

[10] Yang, H., Zhou, Y., Hu, Y. H., Wang, B., \& Kung, S. Y. (2018, May). Cross-layer design for network lifetime maximization in underwater wireless sensor networks. In 2018 IEEE International Conference on Communications (ICC) IEEE. Doi: 10.1109/ICC.2018.8422176

[11] Ahmed, S.H.; Lee, S.; Park, J.; Kim, D.; Rawat, D.B. iDFR: Intelligent directional flooding-based routingprotocols for underwater sensor networks. (CCNC), Las Vegas, NV, USA (2017); doi.org/10.1109/ccnc.2017.7983168

[12] Vijayalakshmi, P.; Noorunnisa, N.; Rajendran, V. Performance analysis of $\mathrm{VBF}$ protocol for position tracking of moving nodes in underwater communications. In Proceedings of the 2017 International Conference (ICCSP), Wuhan, China, 1719 March 2017; doi:10.1109/iccsp.2017.8286708

[13] Khasawneh, A.; Latiff MS, B.A.; Kaiwartya, O.; Chizari, H. A reliable energy-efficient pressure-based routing protocol for underwater wireless sensor network. Wirel. Netw. 2017, 24, 2061-2075, doi:10.1007/s11276-017-1461-x.

[14] Tran-Dang, H.; Kim, D.S. Channel aware cooperative routing in underwater acoustic sensor networks. J.Commun. Netw. 2 019, 21, 33 44, doi:10.1109/JCN.2019.000004.

[15] Daudpota, S. (2019). A Comprehensive Survey on the Performance Analysis of Underwater Wireless Sensor Networks (UWSN) Routing Protocols. International Journal of Advanced Computer Science and Applications, 10(5). https://doi.org/10.14569/ijacsa.2019.0100576. https://doi.org/10.13140/RG.2.2.13755.67363

[16] Ashraf, S., Gao, M., Mingchen, Z., Ahmed, T., Raza, A., \& Naeem, H. 
(2020). USPF: Underwater Shrewd Packet Flooding Mechanism through Surrogate Holding Time. Wireless Communications and Mobile Computing, 2020, 1-12.doi:10.1155/2020/9625974

[17] Wang, Z.; Han, G.; Qin, H.; Zhang, S.; Sui, Y. An Energy-Aware and Void-Avoidable Routing Protocol for Underwater Sensor Networks. Ieee Access 2018, 6, 7792-7801, doi:10.1109/access.2018.2805804.

[18] Hindu, S.K.; Hyder, W.; Luque-Nieto, M.-A.; Poncela, J.; Otero, P. Self-Organizing and Scalable Routing Protocol (SOSRP) for Underwater Acoustic Sensor Networks. Sensors 2019, 19, 3130, doi:10.3390/s19143130.

[19] Barbeau, M.; Blouin, S.; Cervera, G.; Garcia-Alfaro, J.; Kranakis, E. (2015). Location-free link state routing for underwater acoustic sensor networks. In Proceedings of the 2015 IEEE 28th Canadian Conference(CCECE), Halifax, NS, doi:10.1109/ccece.2015.7129510.

[20] Balsamo, S., Marin, A., \& Vicario, E. (Eds.). (2018). New Frontiers in Quantitative Methods in Informatics. Communications in Computer and Information Science. Springer International Publishing. doi:10.1007/978-3-319-91632-3

[21] Balas, Valentina Emilia., et al. Data Management, Analytics and Innovation: Proceedings of ICDMAI 2018, Volume 1. Springer Singapore, 2019.

[22] Liu, X., Liu, P., Long, T., Lv, Z., \& Tang, R. (2018). An efficient depthbased forwarding protocol for underwater wireless sensor networks. 2018 IEEE 3rd International Conference on Cloud Computing and Big Data Analysis https://doi.org/10.1109/icccbda.2018.8386561
[23] Ali, M., Khan, A., Mahmood, H., \& Bhatti, N. (2019). Cooperative, reliable, and stability-aware routing for underwater wireless sensor networks. International Journal of Distributed Sensor Networks. https://doi.org/10.1177/1550147719854249

[24] Coutinho, R. W. L., Boukerche, A., Vieira, L. F. M., \& Loureiro, A. A. F. (2016). Geographic and Opportunistic Routing for Underwater Sensor Networks. IEEE Transactions on Computers, 65(2), 548-561. https://doi.org/10.1109/tc.2015.2423677

[25] Dhurandher, S. K., Obaidat, M. S., \& Gupta, M. (2011). Providing reliable and link stability-based geocasting model in underwater environment. International Journal of Communication Systems, 25(3), 356-375. https://doi.org/10.1002/dac.1245

[26] Aziz, L., \& Aznaoui, H. (2020). Efficient Routing Approach Using a Collaborative Strategy. Journal of Sensors, 2020, 1-17. https://doi.org/10.1155/2020/2547061

[27] Wahid, A.; Kim, D. An Energy Efficient Localization-Free Routing Protocol for Underwater Wireless Sensor Networks. Int. J. Distrib. Sens. Netw. 2012, 8, 307246, doi:10.1155/2012/307246.

[28] Phadke A.G., Thorp J.S. (2017) Electromechanical Wave Propagation. In: Synchronized Phasor Measurements and Their Applications. Power Electronics and Power Systems. Springer, Cham

[29] Qian, L., Zhang, S., Liu, M., \& Zhang, Q. (2016). A MACA-based power control MAC protocol for Underwater Wireless Sensor Networks. 2016 IEEE/OES China Ocean Acoustics (COA). Presented at the 2016 IEEE/OES China Ocean Acoustics (COA). https://doi.org/10.1109/coa.2016.7535810 